La Revue

des Droits

de l'Homme

\section{La Revue des droits de l'homme}

Revue du Centre de recherches et d'études sur les droits fondamentaux

Actualités Droits-Libertés | 2014

\title{
Une évolution du cadre jurisprudentiel du traitement de la demande de report d'audience devant la CNDA
}

Droit d'asile (Cour nationale du droit d'asile)

\section{Cyril Brami}

\section{OpenEdition}

Journals

Édition électronique

URL : http://journals.openedition.org/revdh/856

DOI : $10.4000 /$ revdh.856

ISSN : 2264-119X

Éditeur

Centre de recherches et d'études sur les droits fondamentaux

Référence électronique

Cyril Brami, « Une évolution du cadre jurisprudentiel du traitement de la demande de report d'audience devant la CNDA », La Revue des droits de l'homme [En ligne], Actualités Droits-Libertés, mis en ligne le

30 juillet 2014, consulté le 02 mai 2019. URL : http://journals.openedition.org/revdh/856 ; DOI :

$10.4000 /$ revdh.856

Ce document a été généré automatiquement le 2 mai 2019.

Tous droits réservés 


\title{
Une évolution du cadre jurisprudentiel du traitement de la demande de report d'audience devant la CNDA
}

\author{
Droit d'asile (Cour nationale du droit d'asile)
}

\author{
Cyril Brami
}

1 C'est devenu un lieu commun de présenter la Cour nationale du droit d'asile comme la plus importante juridiction administrative française; ce qu'elle est effectivement, en termes quantitatifs du moins, puisqu'elle aura rendu quelques 38540 décisions en $2013^{1}$. On évoque plus rarement les contraintes, notamment organisationnelles, qui en découlent. Pour parvenir à un tel niveau de "sorties", la Cour organise une dizaine d'audiences par jour, quarante-sept semaines par an, et enrôle quotidiennement plus de deux cents vingt dossiers. Dans ces conditions, alors que le contexte de «crise» du système asilaire français lui impose de raccourcir davantage ses délais de jugement, la question des reports d'audience, qui allongent mécaniquement le délai moyen de la procédure ${ }^{2}$, ne peut manquer d'apparaitre particulièrement sensible. Elle l'est d'autant que le nombre de renvois accordés y est spécialement élevé ${ }^{3}$ au point d'être, selon certains observateurs, "sans équivalent devant les autres juridictions françaises» et qu'on a récemment pu considérer - sans que la méthode d'une telle évaluation soit précisée qu'un tiers de ces renvois n'étaient pas justifiés ${ }^{4}$.

2 Comme en atteste la décision commentée (CE, 18 juin 2014, B. et M., $\mathrm{n}^{\circ}$ 367725), on aurait tort de rechercher les causes de cet état de fait dans l'état du droit du traitement de la demande de report d'audience, tant la jurisprudence apparaît stricte en la matière.

3 Dans l'affaire donnant lieu au pourvoi, la Cour avait été saisie de deux demandes de report d'audience formulées par l'avocat des demandeurs. La première neuf jours avant la tenue de celle-ci, au motif qu'elle avait été fixée à une date correspondant au jour d'indisponibilité hebdomadaire du conseil; la seconde la veille, précisant que les demandeurs, résidant en Isère, ne pourraient se déplacer. Le président de la formation de jugement a refusé de faire droit à ces demandes et donné acte de ce refus dans les visas de 
la décision rejetant au fond les demandes de protection, au motif notamment que leurs « explications écrites se sont révélées insuffisamment personnalisées et concrètes sur (leur) engagement politique (...) et ont paru non crédibles s'agissant des persécutions dont ils auraient été victimes du fait de cet engagement " (CNDA, 30 août 2012, M. et B., nº 11013588 et $\mathrm{n}$ - 11013741, cons. $n^{\circ}$ 5).

4 Saisi en cassation, le Conseil d'Etat fait application des règles du droit commun applicables au traitement des demandes de renvoi par le juge administratif (CE, 16 juillet 2010, C., n ${ }^{\circ} 294239$, au Lebon). La haute juridiction rappelle ainsi l'absence d'obligation pour le juge de faire droit à une demande de report d'audience et consacre une exception, nouvelle en contentieux de l'asile, lorsque sont en jeu les " exigences du débat contradictoire » (cons. $n^{\circ} 2$ ). Constatant qu'en l'espèce, les demandeurs ont été privés de la possibilité d'être assistés par leur conseil du fait de la méconnaissance par la Cour des règles d'enrôlement qu'elle avait elle-même fixées, alors que leur avocat avait formulé en temps utile une demande de renvoi qui n'avait pas de caractère dilatoire, le Conseil d'Etat juge irrégulière la procédure suivie devant la CNDA en raison de ce que les exigences du débat contradictoire imposaient qu'il soit fait droit à la demande de report (cons. $n^{\circ} 4$ )

Ce faisant, le juge de cassation encadre, dans des termes renouvelés, le vaste pouvoir d'appréciation dont dispose le juge de l'asile pour statuer sur la demande de report qui lui est faite $\left(\mathbf{1}^{\circ}\right)$. Il consacre une obligation d'y faire droit, à titre exceptionnel lorsque des motifs tirés des exigences de la contradiction l'imposent, dont il convient de relativiser la portée $\left(2^{\circ}\right)$.

\section{$1 \%$ - L'encadrement prétorien du pouvoir d'appréciation de la demande de renvoi}

6 Sans rompre avec les principes traditionnels gouvernant l'appréciation par le juge de l'asile des demandes de report d'audience formulées devant lui, la décision examinée marque une notable évolution en alignant le régime du report d'audience devant la CNDA sur celui du droit commun (v. CE, 16 juill. 2010, C, préc.). Le Conseil d'Etat prend en effet soin de rappeler que le juge de l'asile, comme tout juge du fond de l'ordre administratif, dispose d'un large pouvoir pour apprécier s'il estime opportun de renvoyer l'examen d'une affaire à une audience ultérieure (v. not. CE, 9 mai 1962, F., Rec. p. 310). On lit ainsi qu'en charge « de veiller à la bonne administration de la justice, [le juge de la CNDA] n'a aucune obligation [...] de faire droit à une demande de report de l'audience formulée par une partie, [et] n'a pas à motiver le refus qu'il oppose à une telle demande » (cons. $n$ ${ }^{\circ} 2$ ).

7 L'affirmation parait conforme aux textes applicables, lesquels octroyent au juge de l'asile un pouvoir discrétionnaire de faire ou non droit à la demande de renvoi. Les dispositions de l'article R. 733-24 du Code de l'entrée et du séjour des étrangers et du droit d'asile (CESEDA) qui habilitent le président de la formation de jugement à statuer sur de telles demandes (al. 4), précisent notamment que «l'absence d'une des parties ou de son avocat n'emporte pas obligation pour (lui) de renvoyer l'affaire » (al. $5^{5}$ ), que son refus n'a pas à être motivé, et qu'il ne peut faire l'objet d'aucun recours (al. 7). La décision de renvoyer ou non l'affaire à une audience ultérieure n'étant pas détachable de la procédure juridictionnelle, elle se présente comme une simple mesure d'administration de la 
justice. Cette dernière peut seulement être invoquée comme un vice affectant la régularité de la décision juridictionnelle statuant sur le fond, dans le cadre du recours contentieux la visant (CE, 7 juin 2000, Z., n² 206362). La liberté du juge est telle qu'il n'est même pas tenu de répondre formellement à la demande de report qui lui est adressée (CE, Ass., 28 oct. 1987, H. H., n 76539, au Lebon).

Comme on sait, cette marge de manœuvre se justifie par le caractère essentiellement écrit de la procédure administrative contentieuse - la CNDA est une juridiction administrative spécialisée et la procédure demeure écrite nonobstant la faculté reconnue aux requérants de présenter des explications orales (v. art. L. 733-1 du CESEDA) - ainsi que par sa nature inquisitoriale - l'instruction étant dirigée par la juridiction, en pratique le président de la formation de jugement et le rapporteur, il lui revient d'apprécier à quel moment l'affaire est en état d'être jugée et de fixer une date d'audience. De tout cela, il ressort que le juge $\mathrm{du}$ fond dispose d'une vaste latitude pour garantir le bon fonctionnement de la justice et empêcher que les parties ne s'arrogent la maîtrise du calendrier de la procédure. Pour autant, ainsi qu'en témoigne la décision examinée, ce large pouvoir d'appréciation qui confine à la discrétionnalité - est en réalité limité de longue date par la haute juridiction. Initialement réservée au cas où la partie qui sollicite le report justifie de circonstances de force majeure (v. CE, Ass., 18 octobre 1980, M. N., n 13914, au Lebon ; CE, Ass., 28 octobre 1987, préc.), l'obligation pour le juge de l'asile de faire droit à cette demande fait ici l'objet d'une notable extension. Le Conseil d'État pose qu'elle trouve à s'appliquer «dans le cas où des motifs exceptionnels tirés des exigences du débat contradictoire l'imposent » (cons. $\left.\mathrm{n}^{\circ} 2\right)$.

9 Si la formulation retenue, littéralement transposée du droit commun (v. CE, 16 juillet 2010, préc.; et CE, 18 octobre 2010, D., n 326020, au Lebon) au contentieux de l'asile, s'intègre probablement dans l'entreprise de "normalisation " de ce contentieux ${ }^{6}$, les prémisses du raisonnement mené par le juge de cassation témoignent de ce que l'obligation consacrée se déduit aussi des caractéristiques propres de la procédure juridictionnelle de détermination.

10 On observe en effet que le Conseil d'Etat articule son raisonnement à partir du texte du premier alinéa de l'article L. 733-1 du CESEDA, lequel impose à la Cour de mettre les requérants à même de présenter leurs observations devant elle et de se faire assister d'un conseil et d'un interprète (v. cons. $n^{\circ} 1$ ). Cette disposition ne fonde certes qu'une simple faculté et non, à rigoureusement parler, un droit absolu pour les parties (jurisprudence constante depuis :CE, 26 juillet 1978, A., nº 06629 au Lebon), elle emporte cependant un ensemble d'obligations pour la juridiction, relatives notamment à la convocation, à l'information des requérants et, désormais, sous certaines conditions, au report de l'audience. En jugeant qu'en l'espèce, le refus opposé à la demande de renvoi méconnaît les exigences du débat contradictoire dans la mesure où il prive les requérants " de la possibilité d'être assistés ou représentés par leur avocat lors de l'audience » (cons. $\mathrm{n}^{\circ} 4$ ), le juge de cassation prolonge l'œuvre jurisprudentielle entamée ces dernières années, qui reconnaît l'importance décisive de l'oralité des débats dans la procédure de détermination ${ }^{7}$. Naturellement, à cet égard, peu importe la nature - juridictionnelle ou administrative - de l'autorité de détermination : il est dans tous les cas illusoire de penser qu'une suite favorable sera donnée à une demande de protection sans que le réfugié ait développé des observations orales - i.e. en réponse aux questions posées par ladite autorité - puisque, pour le dire trop rapidement, la décision sur l'asile implique d'évaluer 
non simplement la cohérence (interne et externe) du récit, mais encore sa crédibilité ainsi que la sincérité de celui qui le formule.

11 La limitation du pouvoir d'appréciation du juge sur la demande de renvoi se présente donc, prima facie, comme une garantie du principe du contradictoire. Pour autant, une lecture serrée de la décision sous examen donne à penser que cette l'obligation est faiblement contraignante.

\section{$2 \%$ - La faible portée contraignante de l'obligation de faire droit à la demande de report}

12 Le taux de renvoi devant la CNDA, dont on a rappelé le niveau élevé, a été récemment dénoncé comme l'une des sources de l'allongement des délais de jugement devant la juridiction ${ }^{8}$ A cet égard, la décision sous examen, malgré l'obligation nouvelle qu'elle fait peser sur le juge de la CNDA, ne devrait pas avoir pour effet d'accroître cette proportion d'affaires renvoyées. Outre que la violation de l'obligation considérée résulte ici de la nécessité pour la Cour de respecter les règles d'enrôlement qu'elle se fixe elle-même (A), les conditions posées par le juge de cassation prémunissent de toute automaticité dans son application (B).

\section{A - Une obligation fondée sur le nécessaire respect par la CNDA de ses propres règles d'enrôlement}

En l'espèce, le Conseil d'Etat fait application du principe issu de la jurisprudence Colomb à un cas dans lequel la Cour n'a pas respecté le dispositif qu'elle " a mis en place, à la fin de l'année 2011, (...) permettant notamment aux avocats d'indiquer, s'ils le souhaitent, pour toute la durée de l'année civile, un jour fixe hebdomadaire pendant lequel ils ne seront pas convoqués devant les formations de jugement » (cons. $\mathrm{n}^{\circ} 3$ ). Ce dispositif, élaboré « en concertation avec les représentants du Conseil national des barreaux et des barreaux concernés, dans le but d'améliorer l'inscription au rôle des affaires et de mieux organiser la convocation des avocats à l'audience " (même cons.), se présente comme un engagement de la juridiction auprès des avocats. Sa méconnaissance, sans justification tirée d'une bonne administration de la justice, viole les exigences de la contradiction puisque les requérants ont "été privés de la possibilité d'être assistés ou représentés par leur avocat lors de l'audience» (cons. $\mathrm{n}^{\circ} 4$ ), et constitue l'un des "motifs exceptionnels" imposant de répondre favorablement à la demande de report (cons. $n^{\circ} 2$ ). La contrainte qui résulte d'une telle obligation pour la juridiction ne doit cependant pas être surévaluée car le système d'organisation des audiences élaboré par la Cour peut être réformé par elle.

14 La mise en œuvre du «calendrier prévisionnel d'instruction", en application de l'article R. 733-13 du CESEDA, qui institue un mécanisme de pré-convocation des requérants au moins deux mois avant l'audience (dans les faits, la Cour s'est engagée à l'adresser trois mois avant l'appel de l'affaire, la convocation étant quant à elle adressée un mois avant l'audience - R. 733-13 CESEDA), a fourni la première occasion de modifier le régime des indisponibilités déclarées par les avocats. A compter du mois de septembre 2014, celles-ci 
seront réduites de moitié, passant à un maximum de vingt-trois jours d'absences par an, ce qui correspond à une demi- journée d'indisponibilité par semaine. On peut douter qu'une telle révision permette, comme elle le prétend, de renforcer l'effectivité des droits de la défense, même s'il en ressortira une meilleure visibilité sur les convocations dont les parties et leur conseil peuvent être destinataires. Il est notamment permis de penser qu'elle entraînera de sérieuses difficultés pratiques pour ceux d'entre eux qui seront susceptibles d'être convoqués cinq jours par semaine, ne disposant dès lors que d'une demi-journée pour échanger avec les requérants et préparer leur défense. Quoi qu'il en soit de ce dernier point, dans la mesure où l'irrégularité sanctionnée par le Conseil d'Etat résulte, certaines conditions étant satisfaites, de la violation d'un engagement souscrit par la Cour qu'elle peut librement réformer, la contrainte susceptible d'en résulter apparait toute relative. La lecture des conditions posées par le juge de cassation pour que joue cette obligation renforce ce constat.

\section{B - L'absence d'automaticité de l'obligation consacrée}

15 Même dans le cas où la demande de renvoi formulée par l'une des parties trouverait son fondement dans « des motifs exceptionnels tirés des exigences du débat contradictoire » (cons. $\mathrm{n}$ - 2), l'obligation d'y faire droit n'est pas automatique. La motivation retenue par le juge de cassation ne laisse pas place au doute : l'obligation est effectivement corrélée à une série de conditions qui préservent, en toute hypothèse, une vaste marge d'appréciation au président de la formation de jugement (v. le cons. $\mathrm{n}^{\circ}$ 4). La décision commentée reflète à cet égard la jurisprudence constante, et traditionnellement stricte, dont la lecture permet de distinguer trois conditions cumulatives qui font systématiquement l'objet d'un examen au cas par cas par le juge de l'asile.

En premier lieu, la demande de report de l'audience ne doit pas être dilatoire (cons. $n$ - 4). Cette condition est appréciée souverainement par le juge du fond, qui doit l'examiner à peine de commettre une erreur de droit ${ }^{9}$. Le juge de l'asile peut ainsi régulièrement refuser de différer l'affaire toutes les fois où il estime que le demandeur a délibérément tenté de placer la Cour dans l'impossibilité de statuer (par ex. en formulant une demande d'aide juridictionnelle la veille de l'audience, CE 8 novembre 2000, B., nº 192470 aux tables; en se présentant à la fin de la séance à laquelle il était convoqué, après le départ de l'interprète et produisant une lettre datée de la veille sollicitant le renvoi de son affaire au cas où la juridiction ne disposerait pas d'interprète, CE 28 avril 2004, A. Q., n - 187078).

17 En second lieu, la demande de report doit être présentée « en temps utile », exigence qui peut recouper la précédente (v. CE, 9 juillet 2001, n 206657). Ainsi les demandes de renvoi formulées la veille ou le jour même de l'audience sont normalement rejetées (v. not. CRR, 13 octobre 1999, n 333030 ; CRR, 8 janvier 1996, n 286921 ; CE, 25 mai 1988, n - 70527) ; le même sort est traditionnellement réservé aux demandes formulées peu de jours avant la tenue de l'audience et motivées par l'insuffisance des délais pour obtenir des éléments de preuve (v. CRR, 23 novembre 1999, n 343759 ; add. CE, 9 juillet 2001, préc. : demande formulée par lettre reçue par la juridiction trois jours avant l'audience).

En troisième et dernier lieu, la demande de renvoi ne doit pas heurter les exigences d'une bonne administration de la justice - ce sera probablement le cas, par exemple, lorsque l'affaire concernée est ancienne et a déjà fait l'objet d'un ou plusieurs renvois, ou encore lorsque sa résolution apparaît très urgente. Soulignons qu'en l'espèce, ce n'est pas 
seulement le fait pour le juge de n'avoir pas respecté le jour d'indisponibilité de l'avocat qui constitue l'irrégularité sanctionnée, c'est de l'avoir convoqué un tel jour sans pouvoir en justifier par un motif tiré d'une bonne administration de la justice. La notion paraît surdéterminer l'ensemble du régime juridique de la demande de report d'audience. En effet, si le juge peut toujours refuser de faire droit à une telle demande au motif que le report contreviendrait au bon fonctionnement de la justice, il doit aussi veiller à ce que son refus ne soit pas contraire à une bonne administration de la justice, laquelle, à lire la décision rendue le 18 juin 2014, prend en compte les engagements souscrits avec les parties afin de garantir la tenue des audiences.

\section{NOTES}

1. V. CNDA, Rapport d'activité 2013, p. 9.

2. Actuellement, le délai moyen constaté s'élève à 8 mois et 26 jours ; v. CNDA, Rapport préc., p. 11.

3. Le taux de renvoi atteint en moyenne $24,2 \%$; v. CNDA, Rapport préc., p. 11.

4. V. le rapport «Létard/Touraine » sur la réforme de l'asile, spéc. p. 56, et l'article $10 \mathrm{du}$ projet de loi relatif à l'asile adopté en conseil des ministres le 23 juillet 2014 ainsi que le dossier de presse officiel.

5. Cette règle ne vaut cependant pas pour les requérants assistés par un avocat désigné au titre de l'aide juridictionnelle, l'absence de l'avocat emportant dans ce cas obligation de renvoyer l'affaire si le demandeur le sollicite.

6. sur quoi, v. AJDA, $\mathrm{n}^{\circ} 41 / 2013$, le dossier intitulé "Actualité du droit des réfugiés ", spéc. S. Slama, «Contentieux de l'asile : l'âge de la maturité ?", p. 2351 et s.

7. Sur le caractère obligatoire, en dehors des exceptions prévues par la loi, de l'audition préalable des demandeurs d'asile par l'OFPRA, v. CE, 3 octobre 2012, CIMADE, n³54995 ; et, CE, 10 oct. 2013, OFPRA contre Y., $n^{\circ} 362798, n^{\circ} 362799$, et nos obs., C. Brami, «Précisions sur le droit processuel applicable à la Cour nationale du droit d'asile », in Lettre "Actualités Droits-Libertés » du CREDOF, 3 décembre 2013.

8. V. entre autres, le rapport « Létard/Touraine » sur la réforme de l'asile, préc..

9. Sur quoi, v. CE, 11 juin 1993, G., n 100093 aux tables ; CE, 29 janv. 2001, B., n 192129 au Lebon. 


\section{RÉSUMÉS}

Dans une décision du 18 juin 2014, le Conseil d'Etat affirme que des motifs exceptionnels tirés des exigences $d u$ débat contradictoire peuvent imposer au juge de l'asile de faire droit à une demande de report d'audience. Il juge irrégulière la procédure suivie devant la Cour nationale du droit d'asile (CNDA) en raison de ce que le refus de différer une affaire enrôlée à tort un jour d'indisponibilité déclarée de l'avocat a eu pour conséquence de priver les demandeurs de la possibilité d'être assistés par leur conseil lors de l'audience sans qu'aucune considération tirée d'une bonne administration de la justice ne le justifie. Cette décision, qui intervient dans un contexte de réforme de l'asile, porte sur une question particulièrement sensible à la CNDA et qui fait régulièrement polémique entre les avocats intervenant régulièrement devant la Cour et la présidence de la CNDA.

\section{AUTEUR}

\section{CYRIL BRAMI}

Maître de conférences en droit public (Université du Maine) 